\title{
ESTRATEGIA PEDAGÓGICA PARA LA FORMACIÓN DE INGENIEROS CON DISCAPACIDAD VISUAL
}

\author{
Flavio Humberto FERNÁNDEZ MORALES ${ }^{1}$ \\ Julio Enrique DUARTE ${ }^{2}$ \\ Guerly José GUTIÉRREZ ${ }^{3}$
}

\begin{abstract}
Resumen
La inclusión educativa de personas en situación de discapacidad es una obligación de las instituciones de educación superior, respaldada por la constitución y las leyes en varios países de Latinoamérica. Aunque se han adelantado esfuerzos para realizar adaptaciones: institucionales, normativas, pedagógicas, curriculares y didácticas, especialmente en los niveles de educación básica y media, son escasas las propuestas didácticas y metodológicas para la formación de ingenieros con discapacidad visual. En el presente trabajo se propone la metodología del aprendizaje basado en problemas, como estrategia didáctica para la inclusión educativa de estudiantes de ingeniería con discapacidad visual. La estrategia se empleó con un estudiante de Ingeniería Electromecánica, invidente a causa de un accidente, quien realizó su proyecto de grado como opción para optar al título profesional. Luego de adquirir las destrezas necesarias para la búsqueda, selección y manejo de la información, fue posible que el estudiante realizara el seguimiento a la solución de un problema en el aula, interactuando con compañeros en una asignatura de octavo semestre. La experiencia fue un reto para estudiantes y docentes ya que no es usual contar en el aula con personas en situación de

\footnotetext{
${ }^{1}$ Doctor en Ingeniería Electrónica, Profesor Titular. Universidad Pedagógica y Tecnológica de Colombia. Carrera 18-calle 23 Duitama, Boyacá-Colombia. flaviofm1@gmail.com

2 Doctor en Física, Profesor Titular, Universidad Pedagógica y Tecnológica de Colombia. Carrera 18calle 23 Duitama, Boyacá-Colombia. julioenriqued1@gmail.com

${ }^{3}$ Licenciado en Ciencias de la Educación y Especialista en Informática para la Docencia, Profesor Asociado, Universidad Pedagógica y Tecnológica de Colombia. Carrera 18-calle 23 Duitama, BoyacáColombia. guerlyjose@hotmail.com
}

Fecha de recepción del artículo: Agosto 2014

Fecha de evaluación: Abril 2015 
discapacidad, esto unido a la escasa orientación que brinda la institución para atender a este tipo de población. Como principal conclusión se resalta la necesidad de brindar capacitación a los docentes universitarios en temáticas referentes a la inclusión educativa, medios didácticos y estrategias pedagógicas alternativas que hagan más efectiva su labor, cuando de trabajar con personas en situación de discapacidad se trata.

Palabras clave: Discapacidad visual, inclusión educativa, formación de ingenieros, aprendizaje basado en problemas

\begin{abstract}
:
Educational inclusion of people with disabilities is an obligation of the institutions of higher education supported by the constitution and laws in several countries of Latin America. Although efforts have been undertaken to make adjustments at institutional, regulatory, educational, curriculum and teaching levels, especially at primary and secondary education, there are still few educational and methodological training proposals for visually impaired engineers. In this paper a methodology of problem based learning as a teaching strategy for inclusive education of engineering students with visual disabilities is proposed. The strategy was used with a blind student of Electromechanical Engineering, caused by an accident, who developed his graduation project to get his professional degree. After acquiring the skills needed for the search, selection and management of information, it was possible for the student to undertake the monitoring of a problem solving in the classroom when interacting with peers in a subject called research seminar of the eighth semester. The experience was a challenge for students and teachers since it is unusual to have disable people in the classroom, along with the limited guidance provided by the institution to help this type of population. As a main conclusion, the need for training university teachers on topics related to inclusive education, teaching aids and alternative teaching strategies is highlighted to make their work more effective when working with this type of disable people.
\end{abstract}

Keywords: Visual disability, inclusive education, engineering education, problembased learning

\title{
Resumé
}

L'insertion scolaire des personnes handicapées est une obligation pour les établissements d'enseignement supérieur, soutenus par la constitution et les lois dans plusieurs pays d'Amérique latine. Bien que des efforts ont été entrepris pour faire des ajustements: institutionnel, réglementaire, éducatif, les programmes et l'enseignement, en particulier dans les niveaux de l'enseignement primaire et secondaire, il y a peu de formation pédagogique et méthodologique pour des ingénieurs ayant une déficience visuelle. Dans cet article, la méthode de l'apprentissage par problèmes est proposé, comme une stratégie d'enseignement pour l'éducation inclusive des élèves ingénieurs ayant une déficience visuelle. La stratégie a été utilisée avec un étudiant d'ingenierie électromécanique, aveugle à cause d'un accident, qui a développé son projet de cessation d'études comme une option pour choisir le titre professionnel. Après avoir acquis les compétences nécessaires à la recherche, la sélection et la gestion de l'information, il a été possible pour lui effectuer un suivi de la résolution du problème en 
classe, interaction avec les partenairs dans un sujet de recherche du huitième semestre. L'expérience a été un défi pour les étudiants et les enseignants, car il n'est pas habituel dans la salle de classe avoir des personnes handicapées, ceci couplé avec le peu d'indications fournies par l'institution pour servir cette population. La principale conclusion met en évidence la nécessité de former des professeurs d'université sur des sujets liés à l'éducation inclusive, outils pédagogiques et des stratégies d'enseignement alternative à rendre leur travail plus efficace, quand il s'agit de travailler avec des personnes handicapées.

Mots-clés: Déficience visuelle, l'éducation inclusive, la formation des ingénieurs, l'apprentissage par problèmes

\section{Introducción}

La inclusión educativa de personas en situación de discapacidad es una obligación de las instituciones educativas, situación que ha sido liderada por organismos multilaterales como la Organización de las Naciones Unidas para la Educación, la Ciencia y la Cultura, UNESCO, y cuyos principios se han expresado a través de diversas declaraciones, conferencias e informes (Parra, Pasuy \& Flórez, 2012). El objetivo de éstos procesos de inclusión es la mejora de la calidad de vida de las personas con discapacidad, favoreciendo aquellos factores personales y ambientales que impacten positivamente su nivel de vida, así como las relaciones laborales y familiares de éste grupo poblacional (Henao \& Gil, 2009).

Lo anterior implica cambiar la visión asistencialista con que la sociedad usualmente ha tratado a los discapacitados, limitando sus posibilidades, por otra en la cual ellos puedan asumir su propio destino con autonomía (Picolo \& Mendes, 2013). Es así como los sistemas educativos en varios países de Latinoamérica buscan generar propuestas educativas incluyentes, que garanticen procesos efectivos de aprendizaje, acceso, permanencia, promoción y evaluación para las personas con Necesidades Educativas Especiales, NEE (Fabela \& Robles, 2013).

Las estrategias de acceso y retención de estudiantes con NEE pasan por elementos comunes como la eliminación de barreras al medio físico y las adaptaciones arquitectónicas, así como por el apoyo económico a través de becas, buscando incrementar los índices de acceso, permanencia y egreso exitoso de las personas con NEE. Sin embargo, son menos frecuentes temas de gran importancia para la inclusión educativa como: las adaptaciones curriculares, garantías de pleno acceso a la información de los programas y asignaturas, los ambientes de aprendizaje, las formas y diferenciación en la evaluación, los acompañamientos, así como la formación inicial y contínua de los docentes para asumir el reto de la inclusión académica en el aula (Tenorio, 2011; Espinosa, Gómez \& Cañedo, 2012).

La inclusión académica de personas con discapacidad visual se ha desarrollado principalmente en los niveles de educación preescolar, básica y media, a través de adaptaciones curriculares y la incorporación de material didáctico especializado (Morgado \& Ferreira, 2011). También se han llevado a cabo investigaciones que buscan aprovechar la experiencia educativa de los estudiantes con discapacidad visual, vivenciada en los niveles básicos de formación, para su vinculación exitosa a la 
educación superior (Ríos \& Moreno, 2013). Aquino, García \& Izquierdo (2012), en un estudio de caso establecieron el grado de visión como un factor relevante en la trayectoria académica de estudiantes con discapacidad visual, así como la necesidad de apoyos institucionales en tres categorías: accesibilidad arquitectónica, tecnológica y de personal especializado.

Regiani \& Mól (2013), reportaron la formación de una invidente como licenciada en química, destacando que la educación inclusiva en la universidad debe superar algunas barreras pedagógicas, las cuales no se limitan a la infraestructura física; los docentes apuntaron a la falta de materiales didácticos adaptados, al desconocimiento y la falta de preparación para interactuar con NEE, así como a la poca comunicación entre estudiantes y docentes, como las principales causas del fracaso escolar de personas con discapacidad.

Algunos investigadores han indagado sobre el uso de maquetas táctiles para el estudio de conceptos físicos como: óptica, mecánica, electromagnetismo y física moderna, como herramientas que facilitan la aproximación a significados con alto contenido visual; en ellas se destaca la importancia de contextos comunicativos adecuados, como aspecto central para la participación efectiva de los invidentes en el aula (Pires de Camargo \& Nardi, 2006; Pires de Camargo \& Nardi, 2008; Pires de Camargo et al., 2009).

Otra línea de trabajo ha sido el desarrollo de equipos que faciliten la comunicación y el acceso a la información de personas con discapacidad, como sistemas de lecturaescritura, sistemas de codificación y decodificación de la voz, traductores de lenguaje, herramientas para la representación de información gráfica en relieve y sistemas inalámbricos de asistencia móvil (Hernández \& Jiménez, 2009; Loaiza \& Torres, 2013; Villarroel \& Villarroel, 2014). La informática también ha provisto entornos computacionales que facilitan el acceso a las posibilidades que proveen las Tecnologías de la Información y la Comunicación, TIC, para la inclusión laboral y educativa de personas con discapacidad (Uribe, Jiménez \& Ramírez, 2009; González \& Farnós, 2009). Igualmente se adelantan esfuerzos para impulsar prácticas de diseño inclusivo entre los desarrolladores de sitios WEB, de modo que las páginas de internet cumplan con estándares mínimos de accesibilidad para la población con discapacidad (Moreta \& Rodríguez, 2013).

Aunque se han adelantado esfuerzos para realizar adaptaciones: institucionales, normativas, pedagógicas, curriculares y didácticas, especialmente en los niveles de educación preescolar, básica y media, son escasas las propuestas didácticas y metodológicas para los niveles de educación superior, y aún más escasas las propuestas para la formación de ingenieros con discapacidad visual.

En el presente trabajo se propone la metodología del aprendizaje basado en problemas, ABP, como estrategia didáctica para la inclusión educativa de estudiantes de ingeniería con discapacidad visual.

La estrategia se EMPLEÓ con un estudiante de Ingeniería Electromecánica en la Universidad Pedagógica y Tecnológica de Colombia, invidente a causa de un accidente, quien realizó su proyecto de grado como opción para optar al título profesional. Luego de adquirir las destrezas necesarias para utilizar el computador a través de un programa 
lector de pantalla, lo cual le permitió la búsqueda, selección y manejo de información, fue posible que el estudiante realizara el seguimiento a la solución de un problema en el aula, interactuando con compañeros en una asignatura de octavo semestre.

La experiencia fue un reto para estudiantes y docentes ya que no es usual contar en el aula con personas en situación de discapacidad, esto unido a la escasa orientación que brinda la institución para atender a este tipo de población.

A continuación se describe el contexto en que se llevó a cabo la experiencia, junto con la metodología propuesta para la formación del estudiante con discapacidad visual. Luego se describen los resultados y se realiza la discusión, tomando en cuenta algunos referentes disponibles en la literatura. Finalmente se presentan las conclusiones, resaltando la necesidad de brindar capacitación a los docentes universitarios en temáticas referentes a la inclusión educativa, medios didácticos y estrategias pedagógicas alternativas que hagan más efectiva su labor, cuando de trabajar con personas en situación de discapacidad se trata.

\section{Contexto y metodología}

La experiencia se llevó a cabo durante el año 2012, en el programa de Ingeniería Electromecánica de la Universidad Pedagógica y Tecnológica de Colombia, UPTC, con un estudiante en la fase terminal de su formación académica. El estudiante sufrió una pérdida total de visión a causa de un accidente, permaneciendo alejado cinco años aproximadamente de la universidad. Esta situación le impidió culminar su proyecto de grado, último requisito para finalizar sus estudios. Al retornar a las aulas, el estudiante tuvo que realizar una actualización, consistente en cursar una asignatura regular, a demás de la elaboración del proyecto.

Para orientar el proceso formativo del estudiante, integrándolo al grupo regular, se propuso la siguiente metodología:

Diagnóstico: Consiste en determinar las expectativas del estudiante con respecto a su proceso formativo, los conocimientos previos, las condiciones iniciales a nivel académico, así como las necesidades específicas en cuanto a herramientas y el material de apoyo requerido para lograr los objetivos propuestos. Es decir, se debe hacer un diagnóstico desde el punto de vista académico, establecer los objetivos de la actividad formativa y determinar las herramientas que deben ser apropiadas, tanto por estudiantes como por docentes, para cumplir con las expectativas.

Acompañamiento: En esta etapa el estudiante con discapacidad y el grupo regular se integran para poner en marcha las actividades previstas en el aula. Es indispensable que el docente seleccione una estrategia pedagógica adecuada, como el ABP, que permita el trabajo colaborativo en el aula. Igualmente importante es realizar el seguimiento que permita detectar las fallas y corregirlas en el momento adecuado.

Evaluación: Como parte integral del proceso educativo se sugiere una evaluación formativa, que dé cuenta de los progresos del estudiante. En el caso de la persona con discapacidad, es necesario considerar su adaptación a los materiales y herramientas que 
facilitan el acceso a la información, ya que sin un progreso en su utilización será difícil incorporar los conocimientos requeridos.

\section{Resultados y discusión}

El diagnóstico inicial permitió establecer que el estudiante no tenía experiencia previa en el uso del computador. Considerando el conocimiento del docente sobre ayudas tecnológicas para invidentes, se le sugirió al estudiante trabajar con el programa JAWS for Windows, software lector de pantalla que verbaliza la información presente en la pantalla de un computador, permitiendo que el usuario acceda a las aplicaciones de uso general, así como a programas de uso específico (Freedom Scientific, 2014). Con ésta herramienta es posible trabajar la ofimática, manipulando archivos de texto en diferentes formatos, hojas de cálculo y presentaciones; también se brinda el acceso a internet, abriendo así la posibilidad de utilizar el correo electrónico, navegar por sitios institucionales y comerciales, consultar revistas especializadas y acceder a todo tipo de información útil en el proceso formativo de cualquier persona.

Es importante realizar el diagnóstico del tipo y grado de discapacidad, para así poder determinar las ayudas más adecuadas a cada situación. En el caso de la vista se puede tener baja visión, donde se conserva un residuo útil para acceder a información gráfica, en cuyo caso es aconsejable un software magnificador de pantalla; cuando la pérdida de la visión es total, se debe emplear un software lector de pantalla. En Colombia el Ministerio de Tecnologías de la Información y las Comunicaciones, MINTIC, provee de forma gratuita la licencia de los programas JAWS y MAGIC, como herramientas que permiten éste tipo de funcionalidad, garantizando que las personas con discapacidad puedan acceder a las posibilidades que brindan las tecnologías de la información y la comunicación (MINTIC, 2014).

La tecnología también provee escáneres que digitalizan información impresa y la convierten en archivos que pueden ser verbalizados; de ésta manera es posible que un discapacitado visual acceda de forma autónoma a información impresa en libros, periódicos y revistas. Igualmente están disponibles las llamadas líneas Braille, que convierten a éste código la información presente en la pantalla del computador, así como las impresoras Braille que permiten al invidente capacitado en éste sistema, acceder y procesar la información según sus necesidades (Isaila, 2014).

El trabajo en el aula se desarrolló bajo la metodología ABP, para lo cual el estudiante discapacitado elaboró un banco de problemas a ser utilizado en el grupo regular. Un requerimiento importante de los problemas propuestos era que reflejaran las diferentes áreas de conocimiento del programa de ingeniería electromecánica. Para ello se tuvo en cuenta la experiencia de los docentes de la escuela, quienes fueron indagados con respecto a las temáticas que ellos consideraban más adecuadas para ser desarrolladas bajo la metodología ABP.

El estudiante consultó a los 15 docentes de planta, de las áreas disciplinar y de profundización, por ser ellos los responsables de la formación en temáticas de electricidad, electrónica, mecánica y mantenimiento, propias del Ing. Electromecánico. Desafortunadamente, solo 8 de los 15 docentes respondieron el llamado, lo cual fue una limitante a la hora de reflejar la interdisciplinariedad del programa. 
En éste punto, es preocupante el desinterés de los docentes por colaborar en el proceso formativo de un estudiante con discapacidad visual. Ésta actitud puede obedecer a desconocimiento en el manejo de personas con discapacidad o a la ausencia de políticas institucionales en lo referente a inclusión académica. Como lo afirman Granada, Pomes \& Sanhueza (2013), el docente puede constituirse en una barrera o en un facilitador de las prácticas inclusivas. Además, es importante escuchar a los estudiantes con discapacidad, conocer sus experiencias previas y sus requerimientos formativos, pues ello se convierte en un recurso potente para orientar la actuación del docente en un aula inclusiva (Ríos \& Moreno, 2013).

Teniendo en cuenta que el objetivo de la metodología propuesta es el desarrollo de competencias profesionales en estudiantes con discapacidad visual, integrados al grupo regular, es indispensable que las instituciones de educación superior propongan políticas de inclusión académica, desarrolladas a través de planes que consideren la capacitación de los docentes en prácticas pedagógicas inclusivas.

El trabajo del estudiante consistió en realizar el seguimiento de la aplicación del ABP en un curso regular, vinculándose él mismo con uno de los temas, los cuales fueron seleccionados de las temáticas incluidas en el banco de problemas mencionado anteriormente. En la tabla 1 se presenta la evolución de los problemas trabajados durante un semestre académico, con estudiantes de octavo semestre, en la asignatura de seminario de investigación.

En la asignatura se matricularon 24 estudiantes, lo cual permitió conformar grupos de cuatro personas; la conformación de los grupos fue voluntaria y cada uno de ellos escogió, de acuerdo a su interés, uno de los problemas propuestos. A continuación se lista el título, dado por los estudiantes, de los seis proyectos desarrollados:

P1: Diseño de un sistema de control del nivel de agua para el humedal ubicado en Puntalarga.

P2: Desarrollo de un sistema de adquisición de datos de temperatura en el equipo de condensación tipo gota y película, del laboratorio de transferencia de calor de la UPTC seccional Duitama.

P3: Estudio de las condiciones reales de iluminación y plan de mejoramiento para la UPTC seccional Duitama.

P4: Diseño de una planta móvil para optimizar el proceso de transformación de la piedra caliza en cal para la empresa Calcalisas ingeniería S.A.

P5: Diseño e implementación de un dispositivo electrónico digital para el control de la temperatura de una ducha eléctrica.

P6: Estudio para la solución del problema que tiene la UPTC seccional Duitama frente al robo y ubicación de las bicicletas.

Tabla 1. Evolución de los proyectos a lo largo del semestre. 


\begin{tabular}{|l|l|l|l|l|l|}
\hline CÓDIGO & ANTEPROYECTO & $\begin{array}{l}\text { INFORME DE } \\
\text { AVANCES }\end{array}$ & $\begin{array}{l}\text { INFORME } \\
\text { TÉCNICO }\end{array}$ & $\begin{array}{l}\text { INFORME } \\
\text { FINAL }\end{array}$ & CUMPLIMIENTO (\%) \\
\hline P1 & $\begin{array}{l}\text { Aceptable } \\
\text { Bueno }\end{array}$ & Bueno & Aceptable & $\begin{array}{l}\text { Muy } \\
\text { Bueno }\end{array}$ & 81 \\
\hline P2 & Bueno & $\begin{array}{l}\text { Aceptable } \\
\text { Bueno }\end{array}$ & Bueno & $\begin{array}{l}\text { Muy } \\
\text { Bueno }\end{array}$ & 84 \\
\hline P3 & Aceptable & $\begin{array}{l}\text { Aceptable } \\
\text { Bueno }\end{array}$ & Bueno & Bueno & 80 \\
\hline P4 & Aceptable & Aceptable & Aceptable & Bueno & 72 \\
\hline P5 & Insuficiente & Aceptable & $\begin{array}{l}\text { Insuficient } \\
\text { e }\end{array}$ & Aceptable & 60 \\
\hline P6 & Insuficiente & Aceptable & Deficiente & Aceptable & 51 \\
\hline
\end{tabular}

En la tabla 1, la primera columna corresponde a la identificación de cada proyecto; las siguientes cuatro columnas indican los resultados obtenidos en cada punto de control, valorados cualitativamente de acuerdo con los avances de cada grupo, mientras que la columna 6 muestra el porcentaje de cumplimiento en los objetivos del proyecto; para mayor detalle con respecto a la estrategia empleada se puede consultar a Fernández \& Duarte (2013). El P6 fue el grupo de menor rendimiento debido a que sus integrantes se separaron, en el primer punto de control, por desacuerdo entre ellos.

El estudiante discapacitado participó en el proyecto del humedal, que fue muy interesante pues partió de una problemática concreta. Al tratarse de un problema de su entorno, el estudiante logró interactuar con su comunidad: familia, vecinos y autoridades del lugar, sirviendo de mediador entre la comunidad y los demás integrantes del grupo para la identificación del problema en terreno, la recolección de información de campo y análisis de las posibles alternativas para la solución del problema.

Esta experiencia refleja lo que pueden y deben hacer los estudiantes al enfrentarse a la resolución de un problema real, analizando situaciones y operando sobre ellas, con los conocimientos disciplinares adquiridos y recursos disponibles. De esta manera es posible generar en los futuros ingenieros un aprendizaje significativo, adecuado al desarrollo de las competencias profesionales esperadas en este nivel de formación.

La ventaja de la estrategia pedagógica propuesta radica en que permite el desarrollo de competencias profesionales, lo cual se logra a través de la solución de problemáticas integradoras, que permiten la aplicación de conocimientos previos en la solución de problemas reales. La metodología igualmente favorece la inclusión académica de personas con discapacidad, pues permite que el estudiante aporte sus conocimientos a la solución de un problema, a la vez que se integra a un grupo en condiciones de igualdad. De otro lado, los estudiantes regulares desarrollan una actitud tolerante hacia la discapacidad, reconociendo en las personas con ésta condición fortalezas y habilidades que pueden favorecer el desempeño del grupo de trabajo. 
Con relación al profesor, es necesario que éste descubra quien es su alumno. La inclusión exige que el educador amplíe las competencias que ya posee, como son: investigar, observar, planificar de acuerdo con el alumno que posee, evaluar continuamente su trabajo y su planificación, de modo que pueda tomar los correctivos necesarios en el momento adecuado. Lo anterior es muy importante debido a que la inclusión presupone niveles de individualización de objetivos didácticos compatibles con cada alumno (Souza dos Santos, 2006).

La diversidad en el aula se refleja en estudiantes con diferentes ritmos de aprendizaje, habilidades, intereses, expectativas y necesidades, que demandan la atención educativa adecuada. Esta realidad justifica la necesidad de la formación docente para afrontar los retos de alcanzar el éxito para todos los estudiantes (González et al.,, 2013).

En otras palabras, el docente debe desarrollar competencias que favorezcan el éxito de los procesos inclusivos, en especial la de planificar, diseñar e implantar un currículo integrado e integrador que facilite el aprendizaje significativo de sus estudiantes (Fernández, 2013). Igualmente importante es la capacidad de evaluar el punto de partida del estudiante con NEE, colaborando en la superación de las dificultades que se le presenten. Para ello el docente debe poseer una actitud de autocrítica permanente y ser flexible a la hora de modificar las estrategias pedagógicas que utiliza, pensando siempre en alcanzar los objetivos formativos tanto del estudiante con discapacidad como del grupo regular al cual se encuentra integrado.

La metodología propuesta toma en cuenta competencias básicas que debería poseer todo docente, a saber: la tutoría, entendida como la ayuda continua y dinámica de acompañamiento en la formación y educación del estudiante. La competencia comunicativa, entendida como la capacidad de escuchar, hacer preguntas, expresar conceptos e ideas de forma efectiva. La gestión de metodologías activas, especialmente las colaborativas, pues son ellas las que facilitan la capacitación del estudiante frente al currículo y desarrollan su autonomía. La evaluación formativa, entendida como el seguimiento continuo al avance del estudiante con el fin de corregir las falencias detectadas y promover aquellas estrategias que potencien al estudiante como un todo.

De acuerdo con Vélez (2013), es importante fortalecer la formación docente en tres aspectos: reconocer y trabajar sobre las actitudes de los docentes en formación, brindar los conocimientos, las estrategias y herramientas necesarias para lograr realizar una intervención inclusiva adecuada, y disponer de espacios de práctica donde el docente en formación pueda prepararse para generar procesos inclusivos exitosos.

Para generar docentes proclives a la inclusión académica de personas con discapacidad, es necesaria su formación inicial y continua, a través de cursos que promuevan la formación de actitudes, valores inclusivos, con conocimientos sobre la discapacidad, y con las competencias para generar las adaptaciones curriculares y didácticas que faciliten los procesos formativos de las personas con discapacidad (González et al., 2013). Sin embargo, más importante que las adaptaciones arquitectónicas y tecnológicas, o que los materiales didácticos especiales, para el éxito de las políticas inclusivas se requieren docentes conscientes, con la sensibilidad para adecuar sus prácticas, currículos e instituciones a la diversidad de sus estudiantes. 


\section{Conclusiones}

La inclusión académica de estudiantes con discapacidad se ha convertido en una exigencia para las instituciones educativas de todos los niveles, de modo que las universidades no pueden escapar a ese requerimiento. La responsabilidad de las instituciones de educación superior en garantizar procesos inclusivos es grande, más aún cuando el éxito académico en éste nivel formativo es el que podrá incrementar las posibilidades de los estudiantes con discapacidad de integrarse a la vida productiva y realizarse de manera profesional y personal.

Aunque se han adelantado esfuerzos para realizar adaptaciones institucionales, normativas, pedagógicas, curriculares y didácticas, especialmente en los niveles de educación preescolar, básica y media, son escasas las propuestas didácticas y metodológicas para los niveles de educación superior, y aún más escasas las propuestas para la formación de ingenieros con discapacidad visual.

En el presente trabajo se reportó el uso de la metodología del aprendizaje basado en problemas, como estrategia didáctica para la inclusión educativa de estudiantes de ingeniería con discapacidad visual. La estrategia se EMPLEÓ con un estudiante de Ingeniería Electromecánica en la Universidad Pedagógica y Tecnológica de Colombia, invidente a causa de un accidente, quien realizó su proyecto de grado como opción para optar al título profesional.

La metodología propuesta incorpora tres aspectos para la planificación de actividades de aula inclusivas: diagnóstico de las necesidades educativas, acompañamiento del proceso educativo y una evaluación continua que permita establecer la progresión de los estudiantes, con el fin de ajustar las estrategias en el momento adecuado. Es importante realizar el diagnóstico del tipo y grado de discapacidad, para así poder determinar las adaptaciones curriculares, los materiales didácticos y las ayudas tecnológicas más adecuadas a cada situación.

En éste sentido el docente se presenta como el eje del proceso de inclusión, ya que debe poner en práctica competencias como: la tutoría para el acompañamiento en la formación y educación de los estudiantes, la competencia comunicativa para escuchar y expresar conceptos e ideas de forma efectiva, la gestión de metodologías activas para facilitar el desarrollo de su autonomía, junto con la evaluación formativa para el seguimiento continuo al avance de los estudiantes.

Se destaca la necesidad de brindar capacitación a los docentes universitarios en temáticas referentes a la inclusión educativa, medios didácticos y estrategias pedagógicas alternativas que hagan más efectiva su labor, cuando de trabajar con personas en situación de discapacidad se trata. No basta con la disposición favorable para enfrentar la realidad de un aula inclusiva, y de no contar con los elementos de intervención necesarios, puede llegarse a experiencias poco exitosas.

Las universidades deben asumir su responsabilidad en la inclusión académica de personas con discapacidad y para ello es indispensable que adelanten políticas que 
transformen las instituciones a nivel académico, de infraestructura y de personal, tanto administrativo como docente. Es importante desarrollar planes que consideren la capacitación de los docentes en prácticas pedagógicas inclusivas, conformando equipos interdisciplinares que realicen las adaptaciones curriculares y didácticas, adelanten las asesorías y la evaluación de los procesos de inclusión.

\section{BIBLIOGRAFÍA}

AQUINO, S. P., GARCIA, V. \& IZQUIERDO, J. (2012). La inclusión educativa de ciegos y baja visión en el nivel superior: Un estudio de caso. Sinéctica, 39, 1-21.

ESPINOSA, C. X., GÓMEZ, V. G. \& CAÑEDO, C. (2012). El Acceso y la Retención en la Educación Superior de Estudiantes con Discapacidad en Ecuador. Formación universitaria, 5 (6), 27-38. Recuperado de: http://www.scielo.cl/scielo.php?script=sci_arttextpid=S0718$50062012000600004 \operatorname{lng}=$ estlng=es.10.4067/S0718-50062012000600004.

FABELA, M. A. ROBLES, L. A. (2013). Educación inclusiva y preparación docente: percepciones y preocupaciones de docentes en el aula de educación regular. Simposio internacional: Aprender a ser docente en un mundo en cambio, Barcelona, España, 2122 de noviembre, 2013. Recuperado de: http://som.esbrina.eu/aprender/

FERNÁNDEZ, J. M. (2013). Competencias docentes y educación inclusiva. Revista Electrónica de Investigación Educativa, 15 (2), 82-99. Recuperado de: http://redie.uabc.mx/vol15no2/contenido-fdzbatanero.html.

FERNÁNDEZ, F. H. \& DUARTE, J. E. (2013). EL APRENDIZAJE BASADO EN PROBLEMAS COMO ESTRATEGIA PARA EL DESARROLLO DE COMPETENCIAS ESPECÍFICAS EN ESTUDIANTES DE INGENIERÍA. Formación Universitaria, 6 (5), 29-38.

FREEDOM SCIENTIFIC (2014). Freedom Scientific - Blindness solutions: JAWS. Recuperado de: http://www.freedomscientific.com/Products/Blindness/Jaws.

GONZÁleZ, F., MARTÍN, E., FLORES, N., JENARO, C., POI, R. \& GÓMEZ, M. (2013). Teaching, learning and inclusive education: The challenge of teachers' training for inclusion. 3rd World Conference on Learning, Teaching and Educational Leadership - WCLTA 2012. Procedia - Social and Behavioral Sciences 93 ( 2013 )783-788).

GONZÁLEZ, A. P. \& FARNÓS, J. D. (2009). Usabilidad y accesibilidad para un elearning inclusivo. Revista de Educación Inclusiva, 2 (1), 49-60.

GRANADA, M., POMES, M. P. \& SANHUEZA, S. (2013). Actitud de los profesores hacia la inclusión educativa. Pap. trab. - Cent. Estud. Interdiscip. Etnolingüíst. Antropol. Sociocult. [online]., 25, 51-59. 
HENAO, C. P. \& GIL, L. M. (2009). Calidad De Vida Y Situación De Discapacidad. Revista Hacia la Promoción de la Salud, 14 (2), 112-125. Recuperado de http://148.215.2.10/articulo.oa?id=309126692005.

HERNÁNDEZ, C. A., \& JIMÉNEZ, L. A. (2009). Desarrollo tecnológico para el mejoramiento de la comunicación a distancia entre personas con discapacidad. Revista de Salud Pública, 11 (5), 828-835. Recuperado de: http://www.scielosp.org/scielo.php?script=sci_arttext\&pid=S0124$00642009000500016 \& \operatorname{lng}=$ en\&tlng=es. 10.1590/S0124-00642009000500016.

ISAILA, N. (2014). The assistive software, useful and necessary tool for blind students abilities development. 5th World Conference on Educational Sciences 2013. Procedia Social and Behavioral Sciences 1162014 )2189-2192).

LOAIZA, C. D. \& TORRES, S. J. (2013). Horus: Prototipo de herramienta tecnológica de apoyo semi-libre, para personas con discapacidad visual. Ventana Informática, 29 (2), 13-28.

MINTIC: Ministerio de Tecnologías de la Información y las Comunicaciones, Colombia, (2014). Plan Vive Digital, ConVerTIC. Recuperado de: http://www.vivedigital.gov.co/convertic/.

MORETA, C. \& RODRÍGUEZ, L. (2013). Pautas, métodos y herramientas de evaluación de accesibilidad web. Ventana Informática, 28 (1), 99-115.

MORGADO, F. \& FERREIRA, M. E. (2011). Adaptação de escalas de silhuetasbidimensionais e tridimensionais para o deficiente visual. Revista Brasileira de Educação Especial, $17 \quad$ (1), 21-36. Recuperado de: http://www.scielo.br/scielo.php?script=sci_arttext\&pid=S1413$65382011000100003 \& \operatorname{lng}=$ en\&tlng=pt. $10.1590 /$ S1413-65382011000100003.

PARRA, A. M., PASUY, L. \& FLÓREZ, J. A. (2012). Atención a estudiantes con necesidades educativas diversas: Clave para instituciones de educación superior inclusiva. Plumilla educativa, 10, 126-150.

PICCOLO, G. M. \&MENDES, E. (2013). Contribuições a um pensar sociológico sobre a deficiência. Educação\&Sociedade, 34 (123), 459-475. Recuperado de: http://www.scielo.br/scielo.php?script=sci_arttext\&pid=S0101$73302013000200008 \& \operatorname{lng}=$ en\&tlng=pt. $10.1590 /$ S0101-73302013000200008.

PIRES DE CAMARGO, E. \& NARDI, R. (2006). Planejamento de atividades de ensino de mecânica e física moderna para alunoscomdeficiência visual: dificuldades e alternativas. Rev. electrón. investig. educ. cienc., 1 (2), 39-64. Recuperado de: $<$ http://www.scielo.org.ar/scielo.php?script=sci_arttext\&pid=S1850$66662006000200003 \& \operatorname{lng}=$ es\&nrm $=$ iso $>$.

PIRES DE CAMARGO, E. \& NARDI, R. (2008). Panorama geral das dificuldades e viabilidades para a inclusão do aluno comdeficiência visual em aulas de 
eletromagnetismo. Revista Electrónica de Investigación en Educación en Ciencias, 3 (2), 35-48. Recuperado de: http://www.redalyc.org/articulo.oa?id=273320550004.

PIRES DE CAMARGO, E., NARDI, R., ASSIS DE MIRANDA, N. \& VIZCONDE E. (2009). Contextos comunicacionais adequados e inadequados à inclusão de alunos com deficiência visual em aulas de óptica. Revista Electrónica de Enseñanza de las Ciencias, 8 (1), 93-117.

REGIANI, A. M. \& MÓL, G. D. (2013). Inclusion of a blind student in a chemistry education teachers' undergraduate program. Ciência\&Educação (Bauru), 19 (1), 123 134.

RÍOS, C. A. \& MORENO, K. R. (2013). Processos de escolarização de pessoascomdeficiência visual. Revista BrasileiradeEducação Especial, 19 (1), 61-78. Recuperado de: http://www.scielo.br/scielo.php?script=sci_arttext\&pid=S1413$65382013000100005 \& \operatorname{lng}=$ en\&tlng=pt.10.1590/S1413-65382013000100005.

SOUZA DOS SANTOS, S. (2006). Inclusión, ¿para qué?. Diversitas: Perspectivas en Psicología, 2 (2), 351-359. Recuperado de: http://www.redalyc.org/articulo.oa?id=67920213.

TENORIO, S. (2011). Formación inicial docente y necesidades educativas especiales. Estud. pedagóg., 37 (2), 249-265.

URIBE, G., JIMÉNEZ, J. \& RAMÍREZ, J. (2009). Entorno computacional para el entrenamiento de personas con discapacidad (inclusión laboral y educativa). Revista Educación En IngenieríA, 4 (7), 33-37. Recuperado de: http://www.educacioneningenieria.org/index.php/edi/article/view/80.

VÉLEZ, L. (2013). La educación inclusiva en docentes en formación: su evaluación a partir de la teoría de facetas. Folios, 37, 95-113.

VILLARROEL, F., MARCELO, J. \& VILLARROEL, C. H. (2014). Wireless smart environment in Ambient Assisted Living for people that suffer from cognitive disabilities. Ingeniare - Revista chilena de ingeniería, 22 (2), 158-168. Recuperado de: http://www.scielo.cl/scielo.php?script=sci arttextpid=S0718-

$33052014000200002 \operatorname{lng}=$ estlng=en.10.4067/S0718-33052014000200002. 